\title{
MODELADO, DISEÑO Y CONSTRUCCIÓN DE UN SISTEMA ACTIVO DE CONTROL DE ESTABILIDAD DE BAJO COSTO PARA COHETES EXPERIMENTALES TIPO AFICIONADO
}

\author{
MODELING, DESIGN AND CONSTRUCTION OF AN ACTIVE CONTROL SYSTEM \\ STABILITY OF LOW COST FOR EXPERIMENTAL ROCKETS TYPE AMATEUR
}

\author{
Jorge Alexander Aponte Rodríguez \\ Ing. Mecánico, Docente de la Facultad de Ingeniería Mecatrónica, Investigador Grupo VOLTA \\ Universidad Militar Nueva Granada, Bogotá, Colombia. volta@unimilitar.edu.co
}

\section{Darío Amaya Hurtado}

Ing. electrónico, Docente de la Facultad de Ingeniería Mecatrónica. Investigador Grupo GAV, Universidad Militar Nueva Granada, Bogotá, Colombia. gav@unimilitar.edu.co

\section{Astrid Rubiano Fonseca}

Ing. en Mecatrónica. Docente de la Facultad de Ingeniería programa de Tecnología en Electrónica y Comunicaciones. Universidad Militar Nueva Granada, Bogotá, Colombia. astrid.rubiano@unimilitar. edu.co

Vladimir Prada Jiménez

Ing. en Mecatrónica, Joven Investigador, Facultad de Ingeniería, Investigador Grupo VOLTA Universidad Militar Nueva Granada, Bogotá, Colombia. volta@unimilitar.edu.co

Fecha de recepción: 22 de abril de 2010

Fecha de aprobación: 30 de mayo de 2010

\section{RESUMEN}

Este artículo presenta el modelado, diseño y construcción de un cohete tipo aficionado de bajo costo, empleando un sistema de control activo por medio de una tobera móvil para lograr una mayor estabilidad. Se plantean los métodos y procedimientos utilizados en el experimento, desarrollando el análisis, simulación e implementación de un prototipo de control para lograr una trayectoria de vuelo.

Palabras clave: cohetería, experimental, aficionado, tobera, simulación, modelado, función de transferencia, centro de presión. 


\section{ABSTRACT}

This paper presents the modeling, design and construction of a Rocket amateur of low cost, using an active control system through a mobile nozzle to achieve greater stability. Here describes the methods and procedures used in the experiment, developing the analysis, simulation and implementation of a prototype control for achieve a flight path.

Key words: rocketry, ballistic, experimental, amateur, fan, nozzle, simulation, modeling, transfer function, centre of pressure.

\section{INTRODUCCIÓN}

El desarrollo tecnológico de la cohetería en Colombia, es un tema que ha sido estudiado a nivel académico en algunas universidades. Hace poco la Universidad Sergio Arboleda diseñó, construyó y puso en órbita el primer satélite colombiano: el Libertad Uno, utilizando como medio de transporte el cohete rusoucraniano Dniéper el 17 de abril de 1998 [1].

En universidades como: los Andes [2], [3], [4], [5], [6], (Ingeniería Mecánica) la Universidad de San Buenaventura [7], [8], (Ingeniería Aeronáutica), y la Universidad Nacional de Bogotá (Ingeniería Mecánica) [9], [10], han orientado sus investigaciones al diseño y desarrollo de motores de reacción de propelente sólido; sin embargo, son pocos los trabajos encaminados al control de cohetes, y se considera éste, un tema en constante desarrollo que involucra técnicas de control convencionales [11], [12], y modernas como los algoritmos genéticos, entre otras.

Existen dos aspectos fundamentales que garantizan el seguimiento de una trayectoria de un cohete, propuesta en el planeamiento de la misión; el primer aspecto tiene que ver con la estabilidad (evitar que comience a girar sobre su centro de gravedad), que se logra, ubicando el centro de presiones más cerca de la cola que el centro de gravedad [14], [15]; el segundo aspecto está relacionado con la capacidad del cohete para seguir la trayectoria planeada, lo cual se logra mediante un sistema de control.

Las estrategias de control comúnmente utilizadas en la cohetería, son el control pasivo y el activo. La primera de estas estrategias se caracteriza por ubicar elementos fijos en la aeronave para estabilizar el cohete [11], trabajando adecuadamente cuando se logra un movimiento relativo entre la aeronave y una masa de aire. La segunda estrategia utiliza elementos móviles, capaces de corregir la trayectoria en función de parámetros preestablecidos, involucrando un sistema de control; algunos de los más utilizados son: veletas, aletas movibles, canards, toberas giratorias (empuje vectorial), y motores auxiliares.

En la actualidad la Universidad Militar Nueva Granada en convenio con INDUMIL, está desarrollando un sistema activo de control para lograr la estabilidad de cohetes experimentales tipo aficionado, utilizando sensores y actuadores de bajo costo [12], y generando la apropiación de conocimiento necesaria. Este proceso inició con la obtención 
del modelo estático y dinámico, para así determinar la función de transferencia del sistema por controlar; posteriormente, se diseñó e implementó la estrategia de control, caracterizada por la definición de una posición de referencia con respecto del ángulo de cabeceo, censando una posición real con respecto de ésta, comparándolas y accionando la tobera para minimizar la diferencia entre estas dos, estabilizando el sistema a una referencia seleccionada, para disminuir el error en el sistema. Para llevar a cabo estos objetivos, se utilizaron herramientas de software como son: Matlab (RItool, Simulink), Labview, Proteus (Isis, Ares).

Tabla 1. Convenciones

\begin{tabular}{|c|c|c|c|c|c|}
\hline EJES & DIRECCION & NOMBRE & $\begin{array}{c}\text { VELOCIDAD } \\
\text { LINEAL }\end{array}$ & $\begin{array}{c}\text { PEQUEÑOS } \\
\text { DESPLAZAMIENTOS } \\
\text { ANGULARES }\end{array}$ & $\begin{array}{c}\text { VELOCIDAD } \\
\text { ANGULAR }\end{array}$ \\
\hline OX & ADELANTE & ALABEO & $U$ & $\phi$ & $\mathrm{P}$ \\
\hline OY & ALA DERECHA & CABEZEO & $\mathrm{V}$ & $\theta$ & $\mathrm{Q}$ \\
\hline OZ & ABAJO & GUIÑADA & $\mathrm{W}$ & $\Psi$ & $\mathrm{R}$ \\
\hline
\end{tabular}

\begin{tabular}{|c|c|c|c|c|}
\hline EJES & $\begin{array}{c}\text { MOMENTO DE } \\
\text { INERCIA }\end{array}$ & $\begin{array}{c}\text { PRODUCTO DE } \\
\text { INERCIA }\end{array}$ & FUERZA & MOMENTO \\
\hline OX & $I_{X}$ & $J_{X Y}=0$ & $F_{X}$ & $\mathrm{~L}$ \\
\hline OY & $I_{Y}$ & $J_{Y Z}=0$ & $F_{Y}$ & $\mu$ \\
\hline OZ & $I_{Z}$ & $J_{Z X} \neq 0$ & $F_{Z}$ & $\mathrm{~N}$ \\
\hline
\end{tabular}

\begin{tabular}{|c|l|}
\hline $\begin{array}{c}\text { REPRESENTACIÓN } \\
\text { VARIABLE }\end{array}$ & DESCRIPCIÓN \\
\hline $\mathrm{m}$ & Masa del cohete \\
\hline $\mathrm{U}$ & velocidad lineal a lo largo del eje $\mathrm{X}$ \\
\hline $\mathrm{S}$ & Área de la aleta \\
\hline $\mathrm{q}$ & Presión dinámica \\
\hline$C_{z \alpha}$ & Pendiente de la curva de fuerza normal \\
\hline $\mathrm{\alpha}(\mathrm{s})$ & Ángulo de ataque \\
\hline $\mathrm{Cw}$ & Centro de gravedad, estabilidad y estática longitudinal \\
\hline$\Theta$ & Angulo entre la horizontal y el eje longitudinal de la aeronave. \\
\hline$\theta$ & Variación del ángulo $\Theta$ \\
\hline$\delta$ & $\begin{array}{l}\text { Ángulo de desviación de la tobera con respecto de los ejes de } \\
\text { estabilidad del sistema }\end{array}$ \\
\hline $\mathrm{d}$ & Diámetro del cohete \\
\hline$C_{m \alpha}$ & Deflexión de atraso al momento \\
\hline $\mathrm{l}_{\mathrm{y}}$ & Inercia en el eje y \\
\hline$C_{m \alpha}$ & Estabilidad longitudinal estática \\
\hline$C_{m q}$ & Amortiguación en el cabeceo \\
\hline$C_{m \delta}$ & Inclinación de ascenso \\
\hline $\mathrm{I}$ & Distancia de la base del fuselaje al centro de gravedad \\
\hline
\end{tabular}




\section{METODOLOGÍA}

\subsection{MOVIMIENTOS DEL COHETE}

Con el objetivo de determinar los movimientos del cohete, se ubica un sistema de coordenadas rectangulares unidas a la nave como se ilustra en la figura 1, cuyo punto de referencia es el centro de gravedad de la aeronave.

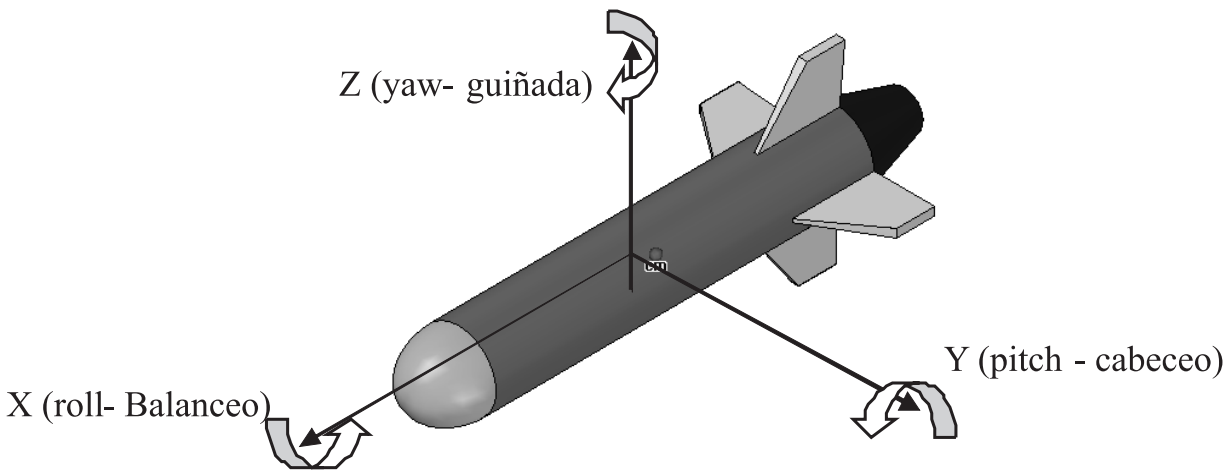

Figura 1. Movimientos de un cohete.

\subsection{FUERZAS EN UN COHETE}

Las fuerzas que actúan sobre un cohete son el empuje, el peso, la resistencia al avance y la sustentación, figura 2. El empuje es la fuerza que genera a partir de los gases de escape del motor, para elevar el cohete. El peso se puede considerar concentrado en el centro de masa del cohete. La resistencia al avance es una fuerza aerodinámica que está directamente relacionada con la geometría del cohete y la altura a la cual se está moviendo. La sustentación depende de la densidad del aire, la velocidad del cohete, la superficie sobre la cual se desliza el aire y su forma e inclinación.

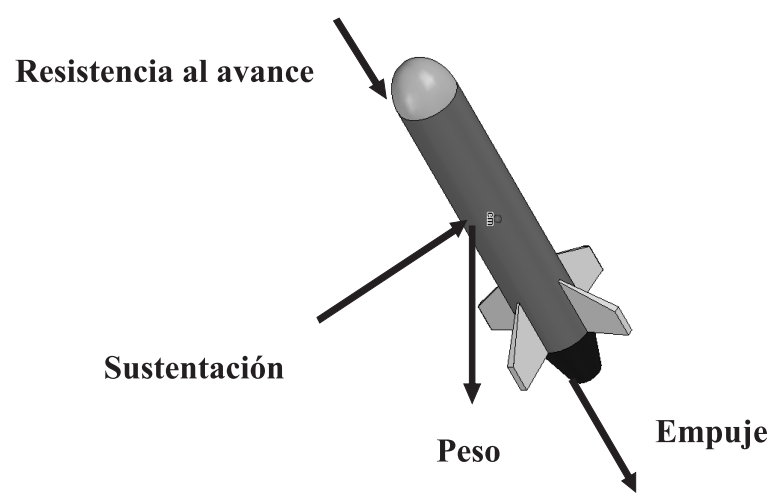

Figura 2. Fuerzas en el cohete. 
Un cohete es considerado estáticamente estable, si una pequeña perturbación a su estado de equilibrio genera fuerzas que tienden a restaurar el equilibrio del cohete. A continuación, se relacionan los aspectos por considerar para lograr la estabilidad de la aeronave:

1. La fuerza de sustentación (lift), es perpendicular a la dirección de vuelo y el arrastre (drag), es paralelo a ésta. Usualmente, el momento de cabeceo es negativo cuando se mide de con respecto del centro aerodinámico.

2. El punto alrededor del cual el momento de cabeceo permanece constante para cualquier ángulo de ataque se denomina centro aerodinámico.

3. El centro de presiones es el punto donde se puede considerar aplicadas todas las cargas distribuidas sobre el cuerpo. En él, concurren la fuerza de sustentación y el arrastre. Normalmente, la localización del centro de presiones queda por detrás del centro aerodinámico y su ubicación varia en función del ángulo de ataque.

4. Cuando la cuerda aerodinámica media del cohete (eje $X$ de equilibrio), se orienta en una dirección diferente al vector de velocidad, aparece el ángulo de ataque ( $\alpha$ ) y con éste, la fuerza de sustentación.

5. La presión dinámica (q) es función de la velocidad y altitud, por lo cual se acostumbra calcular de acuerdo con la velocidad y altitud de la misión.

\subsection{CONSIDERACIONES PARA DETERMINAR LAS ECUACIONES PARA CONTROLAR EL CABECEO (PITCH) Y LA GUIÑADA (YAW)}

Para el análisis, el eje $X$ será el longitudinal del cohete y el sistema de coordenadas asociado a él, será el de estabilidad del sistema. El eje $Z$ se encuentra en el plano de movimiento y el cabeceo se realizará sobre el eje $\mathrm{Y}$, figura 1.

El ángulo a medirá la desviación de la trayectoria con respecto DE la de equilibrio. Para un vuelo en trayectoria curvilínea con cero ángulos de ataque, la aceleración centrípeta debe ser igual a la componente normal al eje $X$ del peso, con lo cual se establece la condición de equilibrio figura 3 a), b) y c). El análisis dinámico se realiza con base en el estudio de perturbaciones a esta condición de equilibrio. 

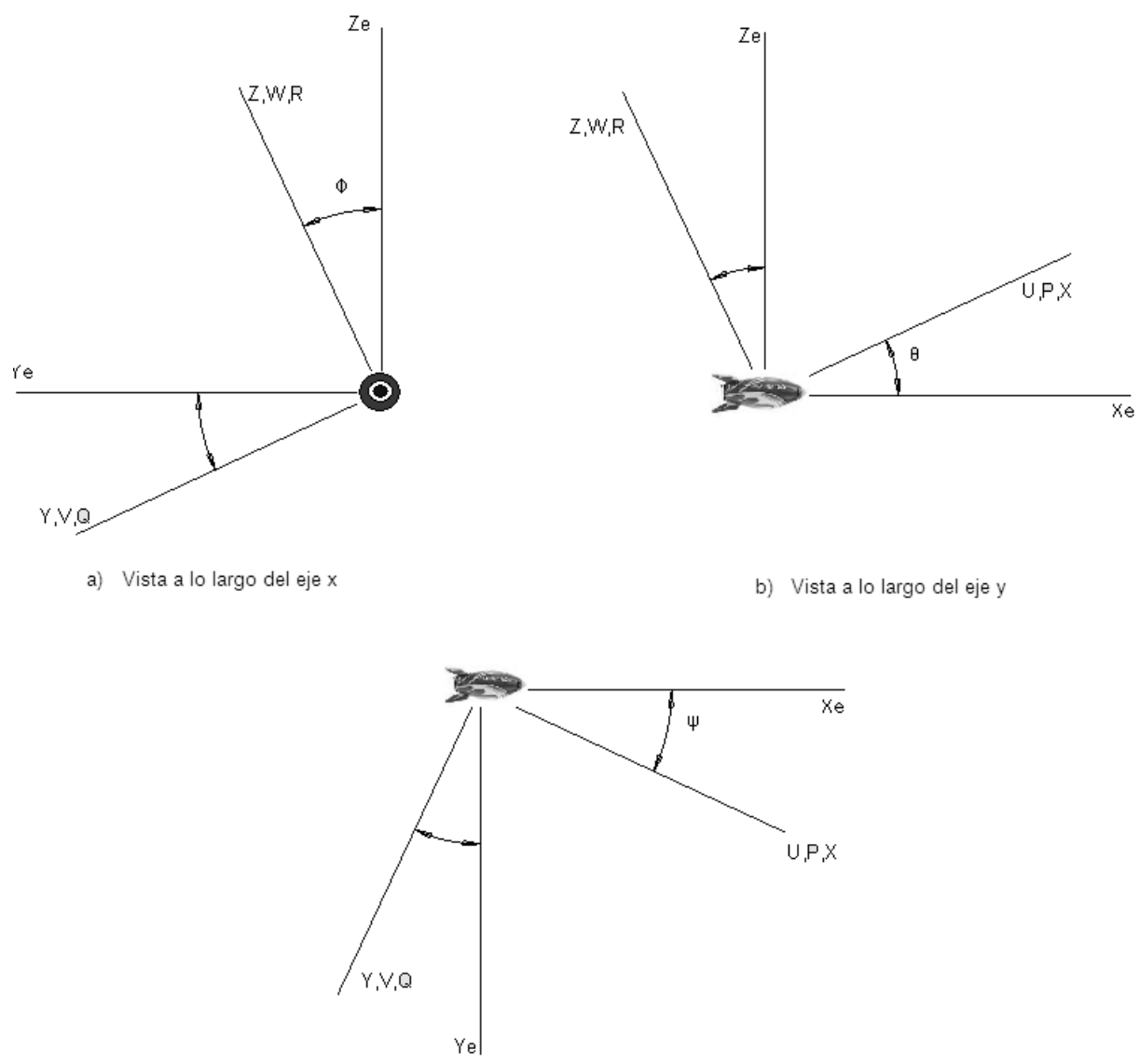

c) Vista Desde el eje z

Figura 3. a), b), c) Sistema de coordenadas del cohete con respectos al sistema de coordenadas de la tierra

\subsection{RESTRICCIONES PARA DETERMINAR LAS ECUACIONES PARA CONTROLAR EL CABECEO (PITCH) Y LA GUIÑADA (YAW)}

El eje X y Z están colocados sobre un plano de simetría y el origen del sistema está localizado sobre el centro de gravedad; la masa del cohete es constante, el cohete es un cuerpo rígido, la Tierra es un sistema inercial de referencia y las perturbaciones son pequeñas. Para efectos de control, la Tierra puede ser tomada como un marco de referencia inercial, particularización que no se podría hacer, si se estuviera intentando realizar algún sistema de guiado. Teniendo en cuenta que las perturbaciones son pequeñas y de corta duración, la velocidad puede ser asumida como constante durante este breve período. 


\subsection{ECUACIONES PARA CONTROLAR EL CABECEO (PITCH) Y LA GUIÑADA (YAW)}

Las ecuaciones que se utilizan para generar la función de transferencia, se determinan asumiendo que las perturbaciones en un cohete son pequeñas, y que su duración es corta. Por lo tanto, la velocidad puede ser asumida constante durante el período de los análisis dinámicos y en un solo eje, es decir, el longitudinal al cohete. Así, las ecuaciones para un período corto se representan en la figura 4 y las ecuaciones de la aeronave son:

$$
\begin{aligned}
& \left(\frac{m U}{S q} s-C_{z_{\alpha}}\right) \alpha_{(s)}+\left(-\frac{m U}{S q} s-C_{w} \sin \Theta\right) \theta(s)=C_{z \delta} \delta \\
& \left(-\frac{d}{2 U} C_{m \dot{\alpha}} s-C_{m \alpha}\right) \alpha(s)+\left(\frac{I_{y}}{S q d} s^{2}-\frac{d}{2 U} C_{m q} s\right) \theta(s)=C_{m \delta} \delta
\end{aligned}
$$

Como no hay un estabilizador horizontal en el cohete el $C_{m \dot{\alpha}}$ es cero.

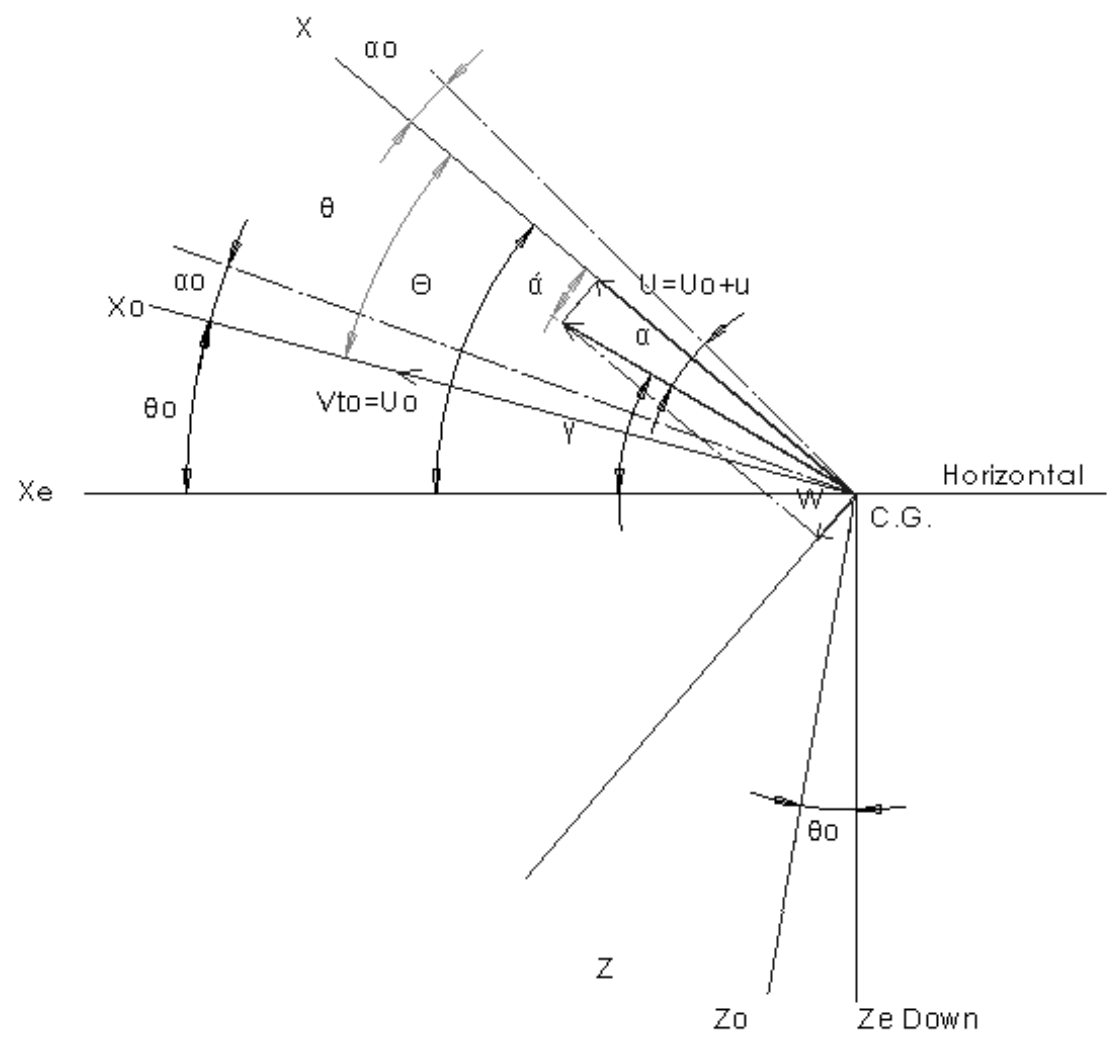

Figura 4. Diagrama de estabilidad del cohete ante perturbaciones 


\subsection{OBTENCIÓN Y ANÁLISIS DE LA FUNCIÓN DE TRANSFERENCIA Y MODELO DEL SISTEMA DE CONTROL}

Para obtener la función de transferencia del cohete, se debe partir determinando los valores de las ecuaciones (1) y (2) y con los siguientes requerimientos de diseño:

$$
\text { Empuje: } 10 \text { N, Velocidad: } 20 \mathrm{~m} / \mathrm{s}
$$

Teniendo estos valores, se procede a determinar las variables que intervienen en las ecuaciones:

$$
\begin{aligned}
& \left(\frac{m U}{S q} s-C_{z_{\alpha}}\right) \alpha_{(s)}+\left(-\frac{m U}{S q} s-C_{w} \sin \Theta\right) \theta(s)=C_{z \delta} \delta \\
& \left(-\frac{d}{2 U} C_{m \alpha} s-C_{m \alpha}\right) \alpha(s)+\left(\frac{I_{y}}{S q d} s^{2}-\frac{d}{2 U} C_{m q} s\right) \theta(s)=C_{m \delta} \delta \\
& m=0.113 \mathrm{Kg}, \mathrm{U}=20 \mathrm{~m} / \mathrm{s}, \mathrm{S}=8.91 * 10^{-4} \mathrm{~m}^{2}, \mathrm{q}=240 \mathrm{~kg} / \mathrm{m}^{2} \\
& C_{z \alpha}=\frac{1}{S q} \frac{\partial F_{z}}{\partial \alpha}=-4,-C_{w}=\frac{m g}{S q}=7.08 \\
& \Theta_{0}=80^{\circ} \\
& \mathrm{C}_{\mathrm{z} \delta}=-\frac{T}{S q}=-46.75 \\
& \mathrm{~d}=0.0337 \mathrm{~m}, \quad-\frac{d}{2 U} C_{m \dot{\alpha}}=0, \quad C_{m \alpha}=\frac{1}{s q d} \frac{\partial M}{\partial \alpha}=-1 \\
& I_{y}=53350 \mathrm{~kg} / \mathrm{m}^{2}, \quad \frac{I_{y}}{S q d}=7.4 * 10^{6} \mathrm{~s}^{2} \\
& \frac{d}{2 U} C_{m q}=-0.00674 \text { segundos } \\
& l=0.14585 m, \quad C_{m \delta}=-\frac{T l}{S q d}=-206.61
\end{aligned}
$$


Substituyendo los valores en las ecuaciones (1) y (2), se obtienen las ecuaciones (3) y (4):

$$
\begin{gathered}
(10.56 s+4) \alpha_{(s)}+(-10.56 s+6.97) \theta(s)=-46.75 \delta \\
(-1) . \alpha(s)+\left(7.4 * 10^{6} s^{2}+0.00674 s\right) \theta(s)=-206.61 \delta
\end{gathered}
$$

A partir de las ecuaciones (3) y (4), la función de transferencia del sistema, teniendo como entrada $\delta$ y salida $\theta$ es (5):

$$
\begin{gathered}
\frac{\theta_{(s)}}{\delta_{(s)}}=\frac{-2181.80(s+0.4)}{78.144 * 10^{6} s^{3}+29.6 * 10^{6} s^{2}-10.48 s+6.97} \\
\frac{\theta_{(s)}}{\delta_{(s)}}=\frac{-2181.80(s+0.4)}{(\mathrm{s}+0.37879)\left(\mathrm{s}-48.785 * 10^{-6}+485.25 * 10^{-6} \mathrm{i}\right)\left(\mathrm{s}-48.785^{*} 10^{-6}-485.25 * 10^{-6} \mathrm{i}\right)}
\end{gathered}
$$

Una vez planteada la función de transferencia, se analiza la estabilidad del sistema mediante el diagrama de polos y ceros, el cual se realiza mediante Matlab, haciendo uso del paquete adicional Rltool; una vez finalizada, se determinó que el sistema es inestable, figuras 5 y 6 .

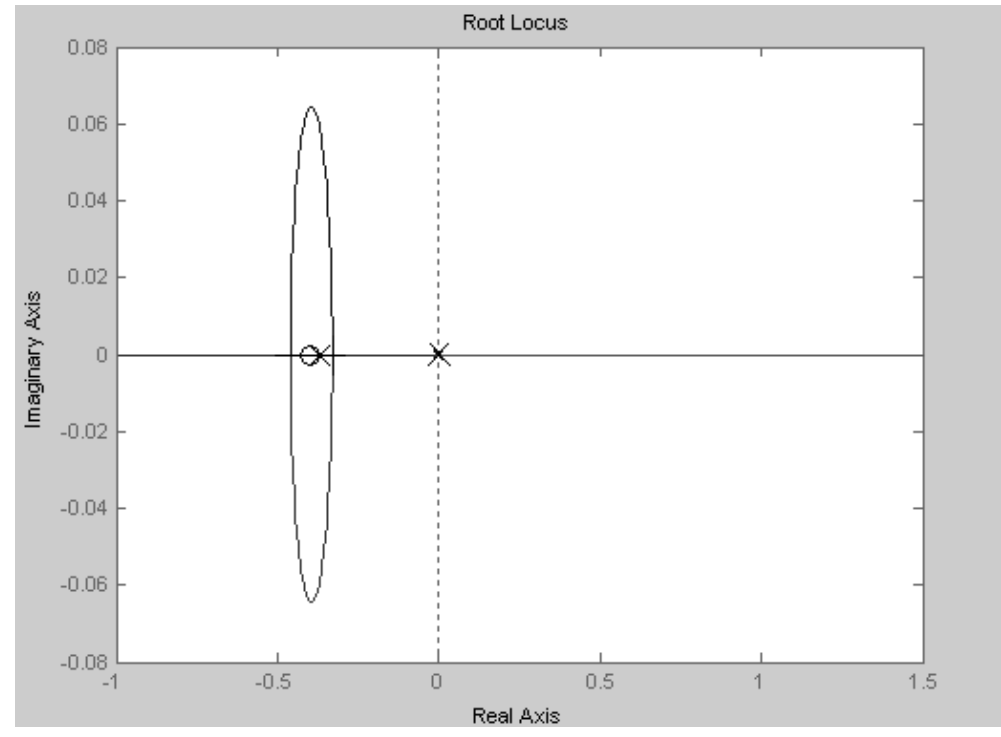

Figura 5. Polos y ceros de la planta. 


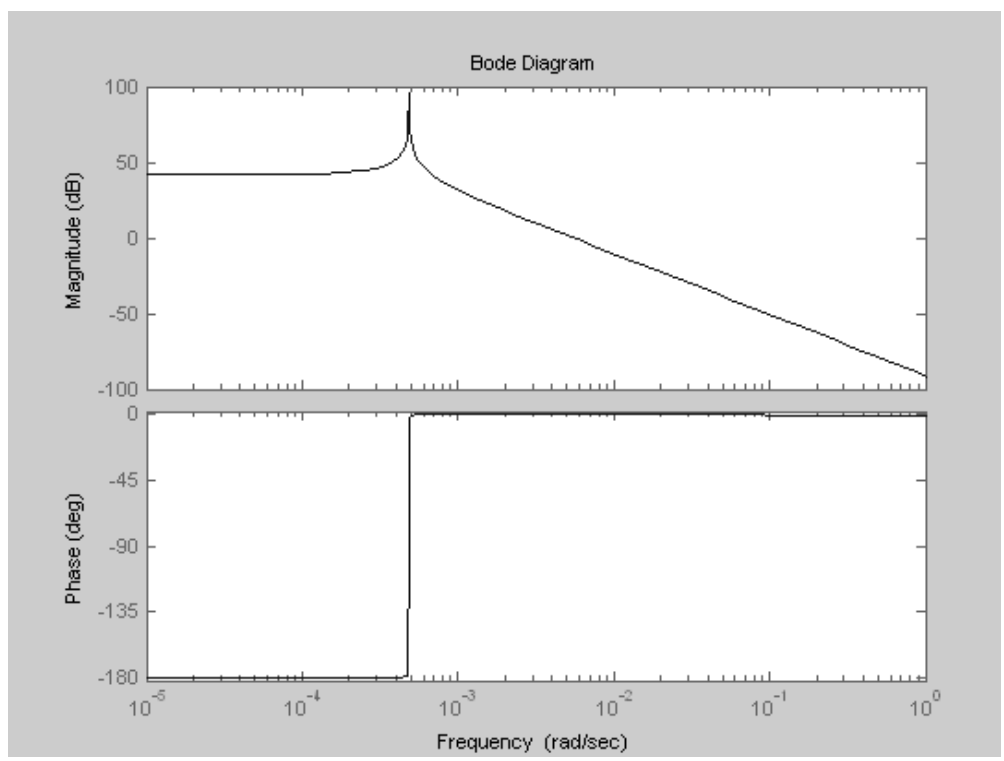

Figura 6. Diagrama de bode de la planta

Concluido el análisis de estabilidad de la planta, se propone implementar un PID, utilizando Simulink $\AA$, que es un paquete adicional de Matlab que permite simular cada uno de los bloques que intervienen en el sistema, y la respuesta de los mismos a diferentes entradas, realizando ajuste a las constantes del controlador para así obtener la respuesta deseada para controlar la posición del actuador.

El diagrama de bloques del sistema completo con el controlador PID, se muestra en la figura 7. En la figura 8, se observa el comportamiento del sistema compensado a una entrada escalón, teniendo como parámetros de diseño un Máximo sobre impulso (Mp) menor al $20 \%$ y un tiempo de asentamiento (ts) menor a $0.8 \mathrm{seg}$ para lo cual las constantes del controlador PID que obtienen son: $k_{p}=1, k_{i}=0.001$, $k_{d}=0.1$

Con estos valores se obtiene la siguiente función de transferencia del controlador:

$$
\begin{gathered}
G_{c}(s)=k_{p} *\left(\frac{1}{k_{i} * s}+k_{d} * s+1\right) \\
G_{c}(s)=\frac{0.0001 s^{2}+0.001 s+1}{0.001 s}
\end{gathered}
$$




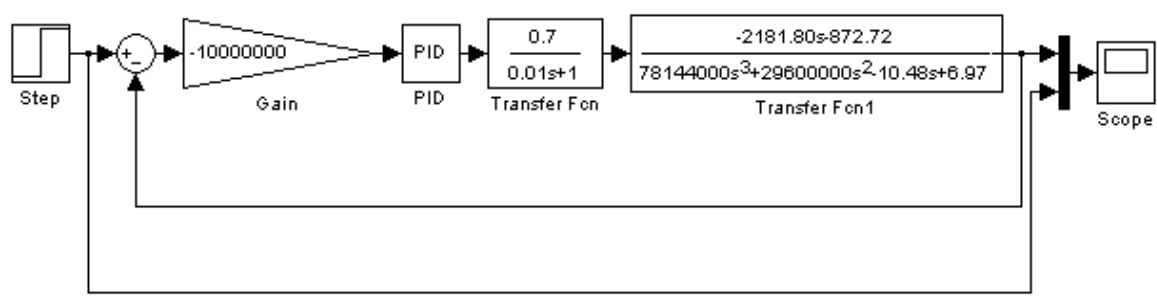

Figura 7. Diagrama de bloques del sistema de control.

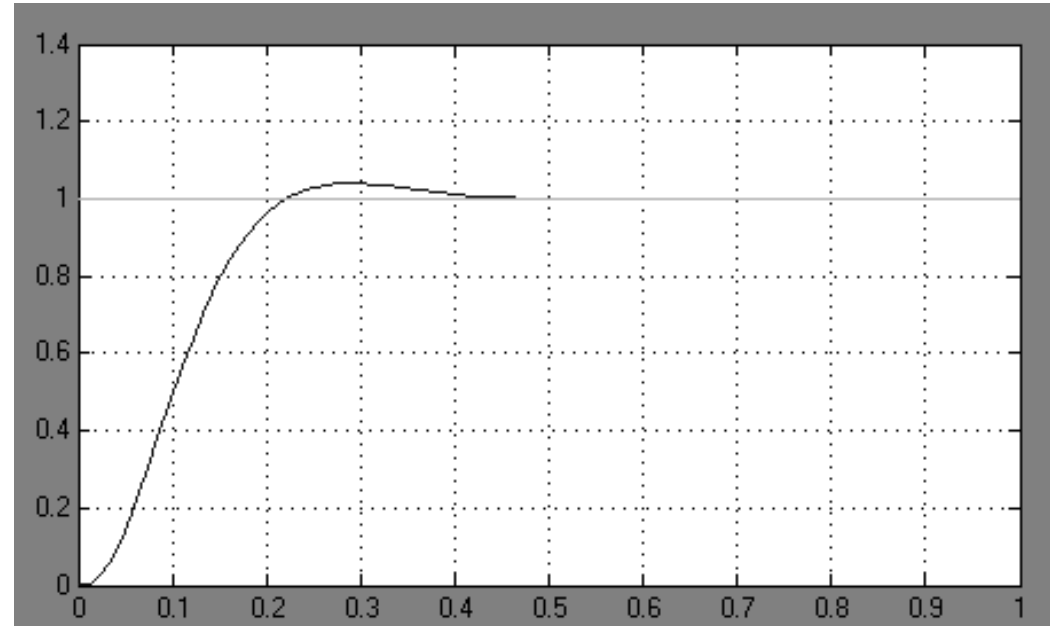

Figura 8. Tiempo de estabilización y sobre impulso resultante del sistema de control.

En la figura 9, se muestra el diagrama de polos y ceros del sistema, y en la figura 10, el comportamiento en frecuencia.

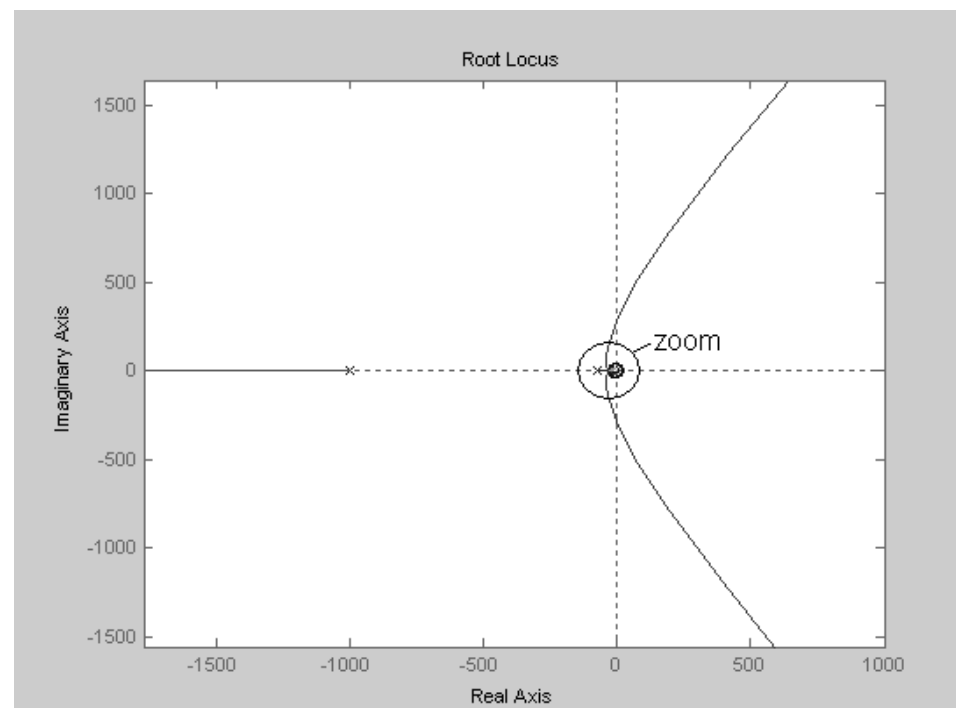

a) Diagrama de polos y ceros del sistema 


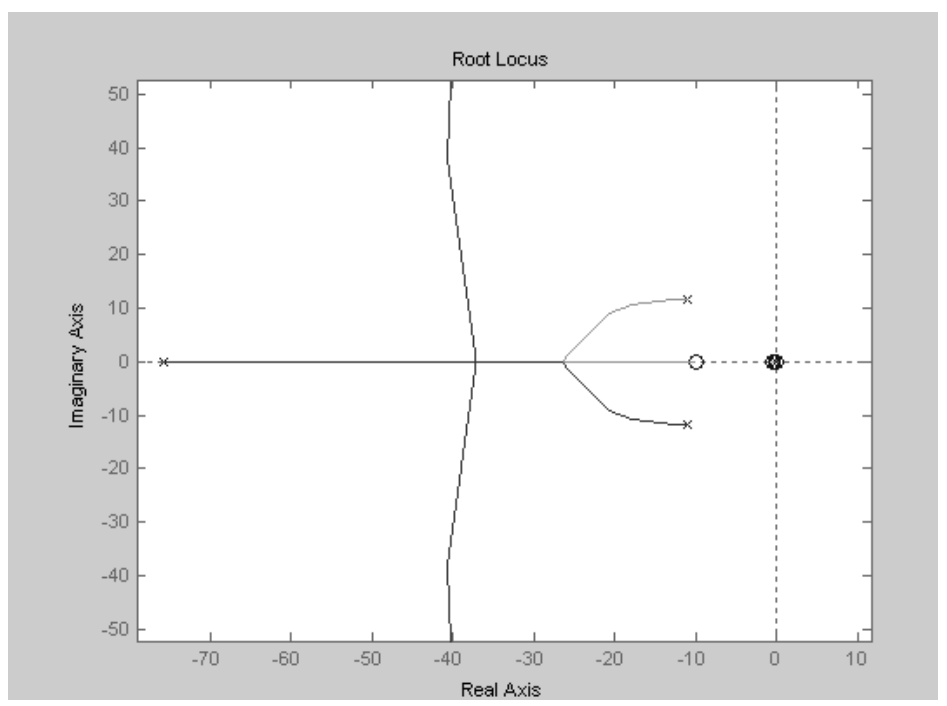

b) Zoom del diagrama de polos y ceros de la Figura a)

Figura 9. Diagrama de polos y ceros del sistema

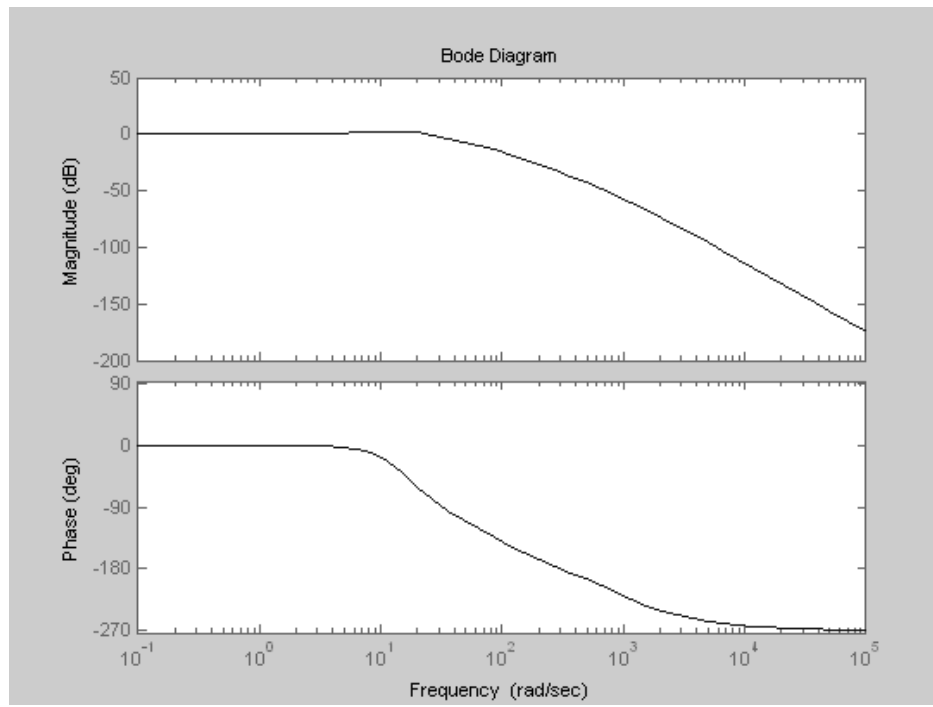

Figura 10. Diagrama de bode del sistema

\section{IMPLEMENTACIÓN DEL SISTEMA DE CONTROL}

\subsection{CONDICIONES DEL SISTEMA DE CONTROL}

Este sistema de control se caracteriza por la definición de una posición de referencia con respecto del ángulo de cabeceo, y permite sensar una posición real con respecto 
de ésta, comparándolas y accionando la tobera para minimizar la diferencia entre estas dos, estabilizando el sistema a una referencia seleccionada para disminuir el error en el sistema. Considerando que el cohete debe girar alrededor de su centro de masa, para realizar el experimento se diseñó un soporte giratorio, figura 11, que permite el movimiento a su alrededor con mínima resistencia.

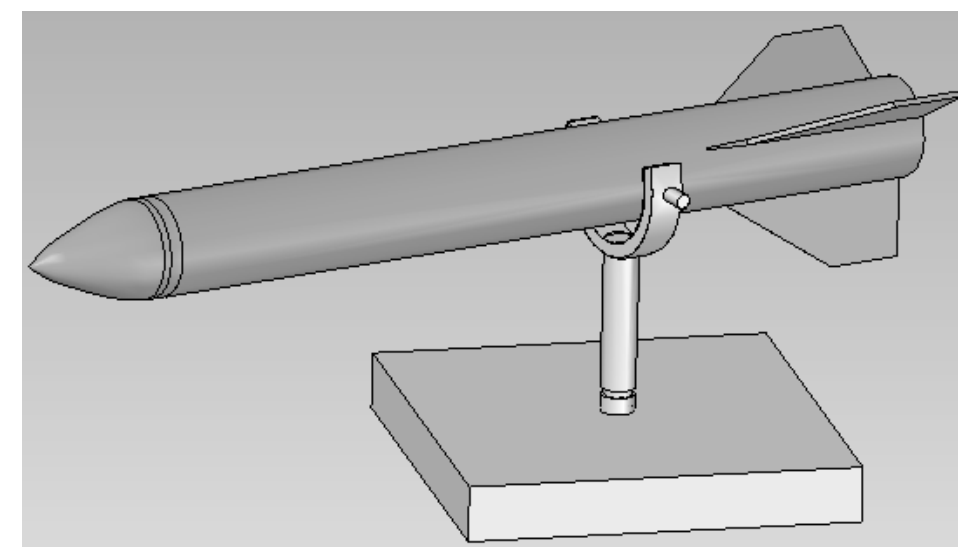

Figura 11. Soporte para realizar el montaje

\subsection{CALIBRACIÓN DEL SENSOR}

Para medir el ángulo de cabeceo, se utilizó un acelerómetro MMA7261QT [16], cuyo principio de funcionamiento es capacitivo y que entrega un voltaje que varía en función a la inclinación sobre los tres ejes $(x, y, z)$. Las variaciones del sistema fueron digitalizadas a 8 bits, mediante un Microcontrolador, determinando así una resolución de conversión de $19.6 \mathrm{mV}$, en un rango de $0 \mathrm{~V}$ a $5 \mathrm{~V}$, considerando que cuando el cohete se encuentra en la referencia, el voltaje entregado por el sensor es de $1621 \mathrm{mV}$ y el número decimal generado por el conversor es 82; este dato se utiliza para determinar el valor absoluto del ángulo entregado con respecto de la posición de referencia.

Mediante un comparador de carátula, se midieron las variaciones del ángulo de inclinación del cohete y a su vez el voltaje, generando por las salidas del sensor y permitiendo desarrollar la curva característica para determinar la resolución del sistema.

Con los datos obtenidos (tabla 1), se realizó la curva de calibración del sistema de medida, figura 12. Con la señal entregada por el sensor, se convirtió de Análogo a Digital, y se empleó el conversor del Microcontrolador con una sensibilidad de 8 Bits; por lo tanto, su resolución de conversión es de 19,6 mV. Teniendo en cuenta que la entrada al conversor cuando se tiene 0 es de $1621 \mathrm{mV}$, y genera un número binario de 82, por lo tanto, la ecuación por resolver en el computador para determinar la posición del cohete es (6): 


$$
0^{\circ}=\left(\frac{\text { Numero Decimal-82 }}{0.94474}\right) * 19.6
$$

Tabla 2. Datos experimentales para la calibración del sensor

\begin{tabular}{|c|c|c|c|c|c|}
\hline \multicolumn{7}{|c|}{$\begin{array}{c}\text { CALIBRACIÓN DEL SENSOR } \\
\text { GRADOS VS VOLTAJE }\end{array}$} \\
\hline GRADOS & $\begin{array}{c}\text { VOLTAJE } \\
(\mathrm{mV})\end{array}$ & MEDIA & $\begin{array}{c}\text { DESVIACION } \\
\text { ESTAND AR }\end{array}$ & ALFA & $\begin{array}{c}\text { INTERVALO } \\
\text { CONFIANZA }\end{array}$ \\
\hline 0,000 & 1621 & 1620,8 & 0,447 & 0,05 & 0,392 \\
\hline 0,623 & 1620 & 1619,4 & 0,548 & 0,05 & 0,480 \\
\hline 1,245 & 1619 & 1618,6 & 0,548 & 0,05 & 0,480 \\
\hline 1,868 & 1618 & 1617,8 & 0,447 & 0,05 & 0,392 \\
\hline 2,490 & 1618 & 1617,6 & 0,548 & 0,05 & 0,480 \\
\hline 3,111 & 1618 & 1617,2 & 0,447 & 0,05 & 0,392 \\
\hline 3,731 & 1617 & 1616,4 & 0,548 & 0,05 & 0,480 \\
\hline 4,351 & 1617 & 1616,2 & 0,447 & 0,05 & 0,392 \\
\hline 4,970 & 1617 & 1615,4 & 0,894 & 0,05 & 0,784 \\
\hline 5,587 & 1616 & 1615,2 & 0,447 & 0,05 & 0,392 \\
\hline 6,203 & 1615 & 1614,4 & 0,548 & 0,05 & 0,480 \\
\hline 12,265 & 1610 & 1609,2 & 0,447 & 0,05 & 0,392 \\
\hline
\end{tabular}

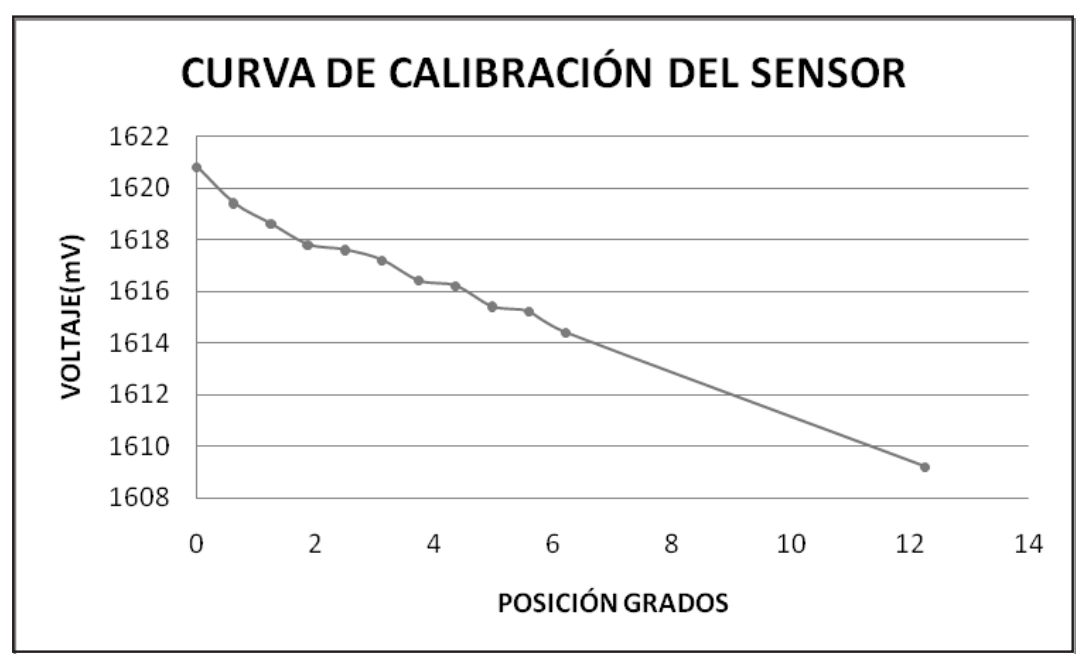

Figura 12. Gráfica de calibración del sensor

El comportamiento del sistema puede ser monitoreado a través de una tarjeta de adquisición de datos que procesa los datos y los envía al computador. 


\subsection{ACTUADORES}

Para el direccionamiento de la tobera, se utilizaron dos servomotores con un voltaje de activación entre 4.8 y $6 \mathrm{~V}$ y un consumo de corriente de aproximadamente $150 \mathrm{~mA}$; del cual se obtuvo como función de transferencia (7):

$$
G(s)=\frac{0.7}{0.01 s+1}
$$

\subsection{SISTEMA MECÁNICO DE ACCIONAMIENTO}

Para logar el redireccionamiento de la tobera, se diseñó y fabricó un sistema mecánico que permite rotar el motor completamente sobre un apoyo tipo rótula, utilizando los dos actuadores descritos anteriormente, ubicados a $90^{\circ}$, como se muestra en la fotografía 1. De esta manera, los servos hacen rotar el motor con respecto de la rótula que a su vez, le da la dirección al cohete, rotando sobre los ejes $Z$ (yaw- guiñada) y Y (pitch cabeceo), descritos en la figura 13.

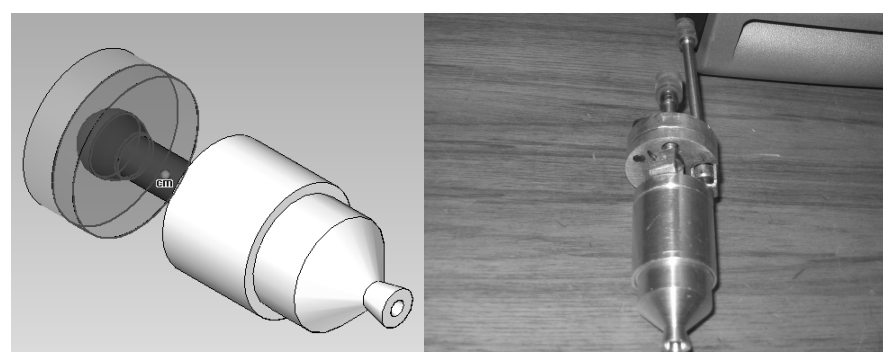

Figura 13. Sistema de posicionamiento del motor

\subsection{ENSAMBLE DEL SISTEMA DE CONTROL}

Como sistema de control, se utilizó un microcontrolador PIC18F2550[17], que procesa las dos salidas análogas entregadas por el acelerómetro. La programación del PIC se realizó mediante el software PIC C COMPILER囚, en el cual se implementó la conversión de análogo a digital con una resolución de 8 bits y la ecuación recursiva equivalente a la digitalización del controlador PID, diseñado para obtener la posición de cabeceo y guiñada, manteniendo la posición de la tobera en un punto central. A continuación, se desarrolló un programa en Labview (figura 14), en donde se encuentran los siguientes bloques: 


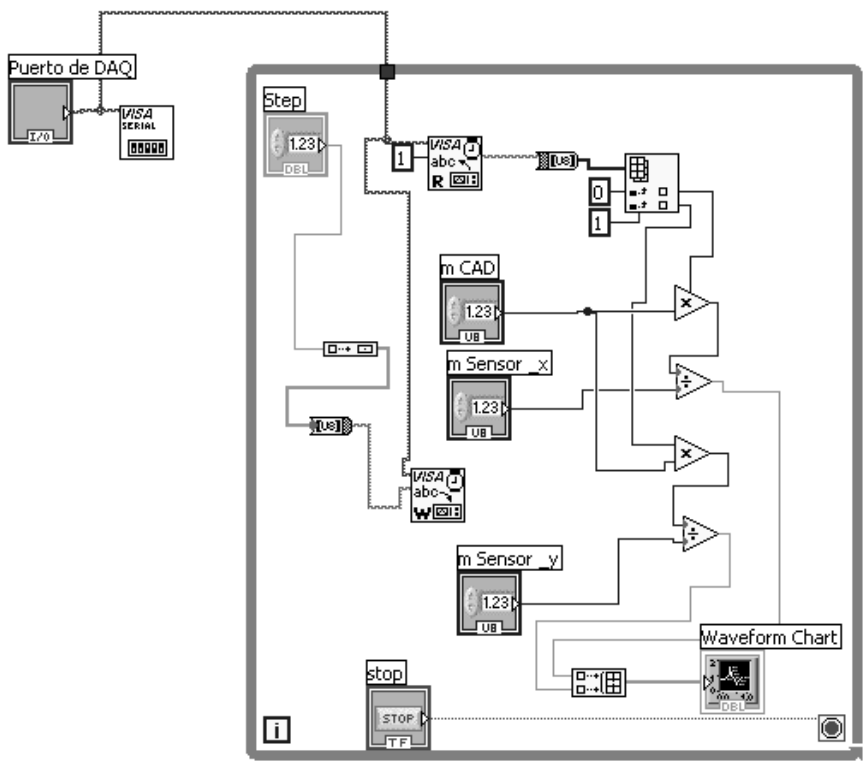

Figura 14. Sistema de monitoreo del sistema de control

1. Puerto DAQ: Comunicación del puerto serial de computador; allí se configura la velocidad de recepción y transmisión.

2. Step: Referencia del sistema, entrada escalón.

3. Visa abc W: Permite visualizar la señal de entrada.

4. M CAD: Corresponde la variable de salida del sistema, que indirectamente controla la variación del PWM para los servomotores.

5. M Sensor $x$ y M Sensor $y$ : Los datos recibidos por el microcontrolador del acelerómetro son digitalizados y enviados mediante protocolo rs232; estos datos corresponden a estas dos variables.

6. VisaR: Corresponde a la entrada en el sistema de visualización de variables; en este caso, corresponde a la salida del sistema que se muestra en la figura 15.

7. Waveform Chart: En este bloque, se visualiza la señal de salida del sistema que es capturada en el bloque VisaR.

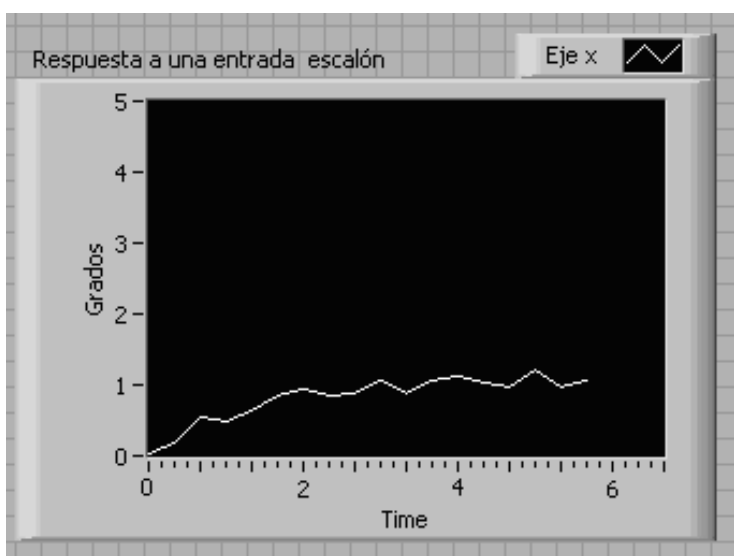

Figura 15. Respuesta del sistema a una entrada de tipo escalón 
La tarjeta electrónica necesaria para la implementación del sistema de control consta de los siguientes elementos: acelerómetro PIC, max-232 y servos representados por el siguiente diagrama de bloque (figura16), y el circuito esquemático diseñado en PROTEUS $®$ (figura17). Para el diseño de la tarjeta, se consideraron las dimensiones del fuselaje del cohete, para lo cual se tiene por separado las tarjetas del acelerómetro PIC y comunicación, como se puede ver en la figura 18.

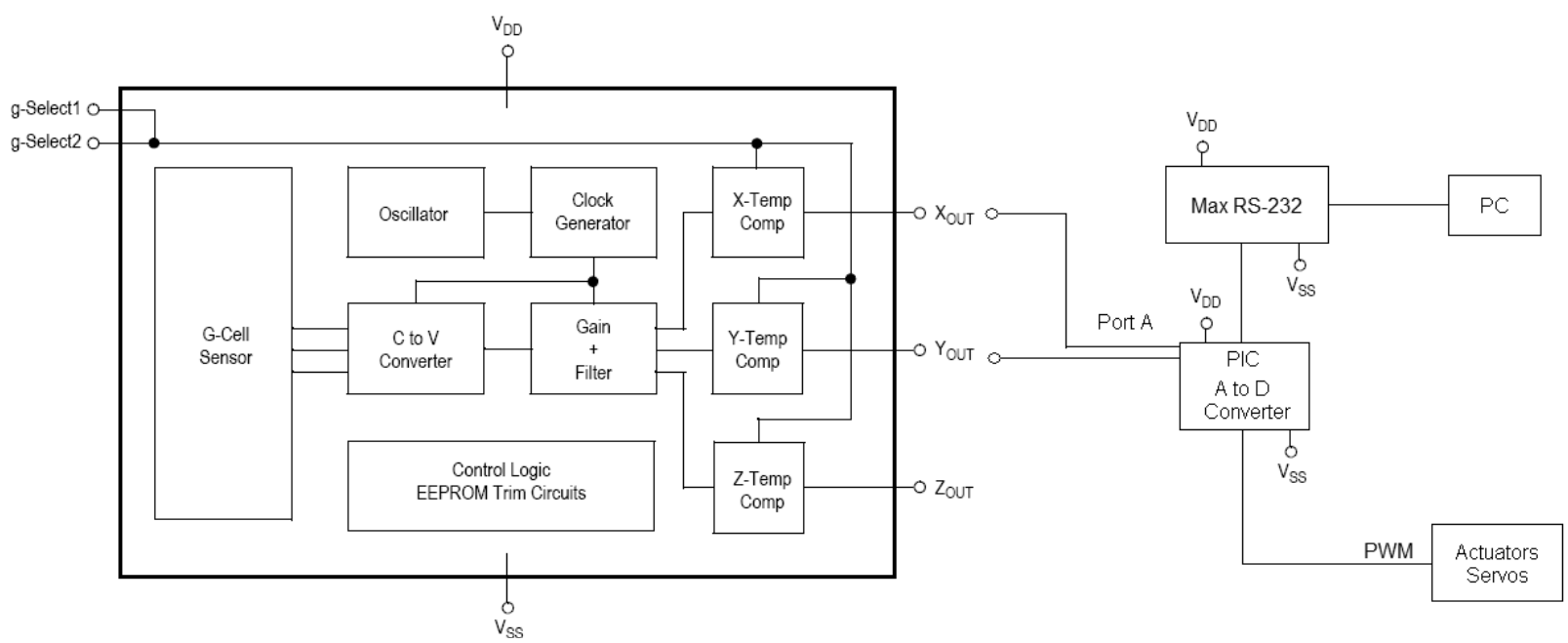

Figura 16. Diagrama de bloques del circuito
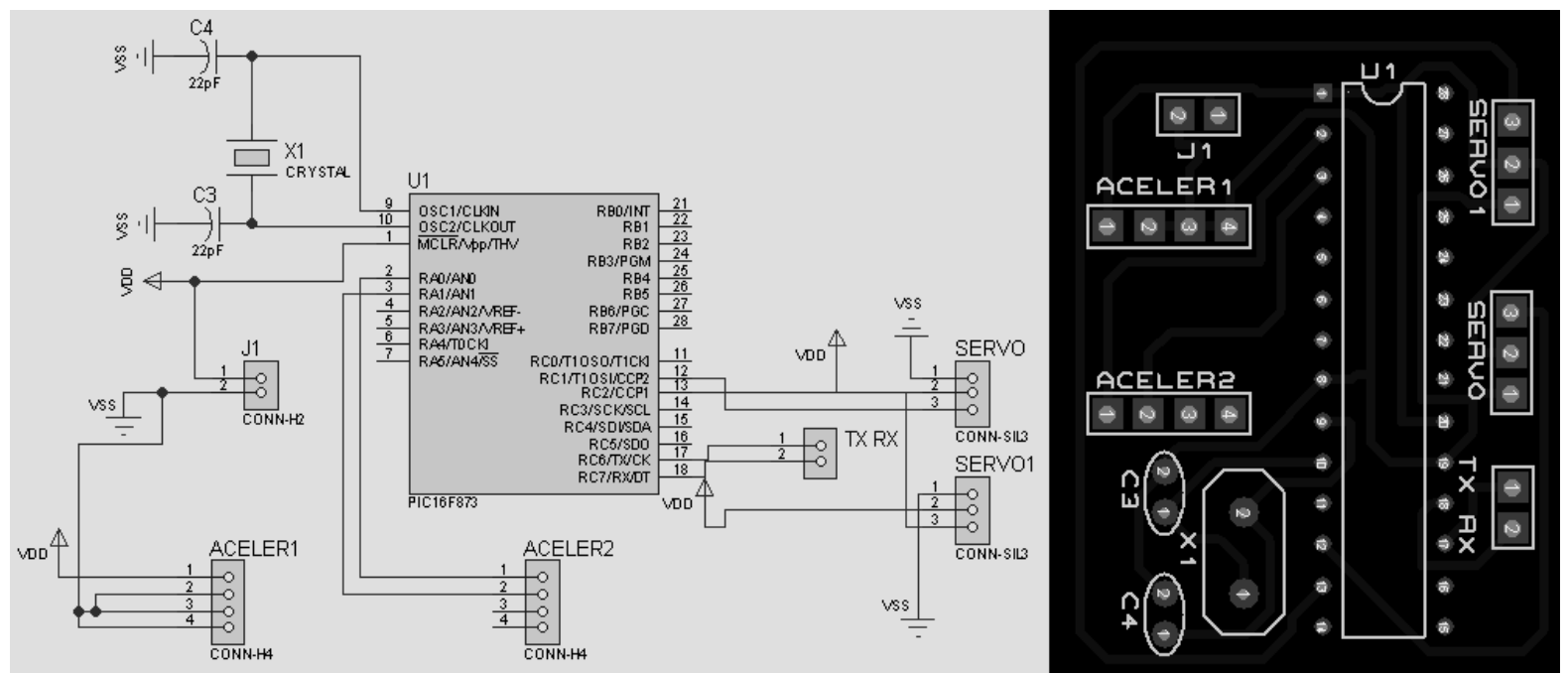

Figura 17. Circuito esquemático y diseño tarjeta 


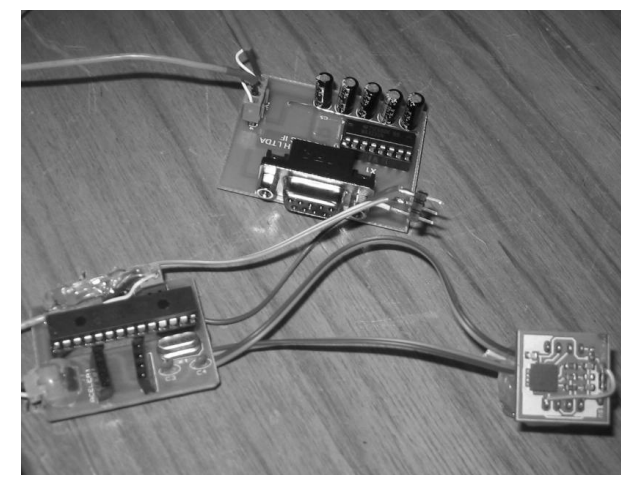

Figura 18. Tarjetas implementadas para el control del cohete

La última etapa consiste en ensamblar la tarjeta electrónica a los sensores y servomotores, los cuales se colocan dentro del fuselaje del cohete (figura 19).

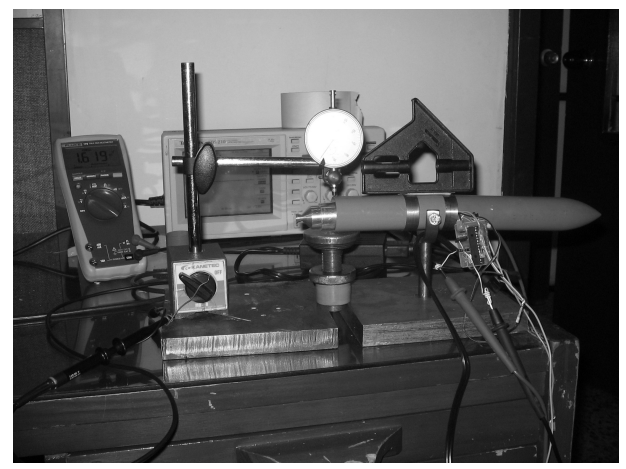

Figura 19. Cohete tipo amateur con el sistema de control activo

\section{CONCLUSIONES}

El modelo de cohete planteado tiene una respuesta del sistema lento para la aplicación; sin embargo, esto puede ser resuelto, utilizando actuadores con mayor velocidad en el tiempo de respuesta, para que el sistema pueda trabajar en tiempos cortos; se logra así un ajuste rápido con relación al cambio de la altitud del cohete. De acuerdo a la sensibilidad, resolución y rango de medición del sensor, se concluye que este es adecuado para la aplicación.

Los cohetes de tipo balístico sin aletas son inherentemente inestables, sin embargo para mejorar el comportamiento del sistema se pueden agregar un tipo de particular de superficies buscando mejorar la estabilidad, para evitar tiempos largos de respuesta. Los elementos fijos que se utilizan para mejorar la estabilidad de un cohete generan tiempos largos de respuesta en sistemas de control activo. 
En futuros trabajos se propone la elaboración de un banco de prueba, que permita evaluar el desempeño de los motores cohete, la elaboración de un prototipo diseñado para alcanzar un apogeo de $1 \mathrm{Km}$, finalmente experimentación con diferentes tipos de combustibles, entre otros trabajos.

\section{REFERENCIAS BIBLIOGRÁFICAS}

[1] Blakelock, J. H. (1990). Automatic Control of Aircraft and Missiles. John Wiley \& Sons Inc, Second Edition, pg 253.

[2] Garzón, A. D. (2002) Análisis y diseño de la Cámara de Combustión de un pequeño Motor Cohete; Bogotá, Trabajo de pregrado (Ingeniero Mecánico). Universidad de los Andes; Facultad de Ingeniería.

[3] Molinares Hernández, L. S. (2007) Construcción de un cohete Amateur Triton II; Bogotá, Trabajo de pregrado (Ingeniero Mecánico). Universidad de los Andes; Facultad de Ingeniería.

[4] Duque Daza; C. A. (2003) Modelo y Caracterización del patrón de flujo en un sistema propulsivo; Bogotá, Trabajo de pregrado (Ingeniero Mecánico). Universidad de los Andes; Facultad de Ingeniería.

[5] Jiménez, A. (2003) Diseño y simulación cohete con carburante sólido; Bogotá, Trabajo de pregrado. Universidad de los Andes; Facultad de Ingeniería.

[6] Aljure Osorio, A. (2007) Construction of a Model Liquid Fueled Rocket Engine; Bogotá, Trabajo de pregrado. Universidad de los Andes; Facultad de Ingeniería.

[7] Bustos Renteria; J. A. (2005) Metodología Matemática para el análisis Aerodinámico de cohetes; Bogotá, Trabajo de pregrado, Universidad de San Buenaventura. Facultad de Ingeniería.

[8] Gomez y Castro (2005) Diseño Preliminar de un Sistema de propulsión líquida con Hidrógeno líquido como Combustible Adaptado a un Rocket Belt; Bogotá, Universidad de San Buenaventura. Facultad de Ingeniería.

[9] Garzón Alvarado, A., Duque Daza C.A. y Roa Garzón M.A. (2004) Introducción general a la tecnología de propulsión; Bogotá, Universidad Nacional de Colombia.

[10] Bustamante, C., Miguel, J. (2003) Micromotor cohete desarrollo de un prototipo exprimental para combustible líquido; Universidad Nacional de Colombia; sede de Bogotá; Facultad de Ingeniería. 
[11] Dragan, V. Lazić and Ristanović, M.R., (May 2007). Electrohydraulic thrust vector control of twin rocket engines with position feedback via angular transducers, Control Engineering Practice, Volume 15, Issue 5, Pages 583-594.

[12] Morita y Kawaguchi (2001). Attitude Control Design of the M-V Rocket, Philosophical Transactions: Mathematical, Physical and Engineering Science.

[13] Tomita, N., Watanabe, R. and Nebylov A.V. (December 2007). Hands-on education system using water rocket. Acta Astronautica; Volume 6.

[14] Barrowman, James. (1966). Century Engeneering Company, INC. Calculating the center of pressure of a model rocket. Disponible en: http://www.estesrockets.com/ images/ uploads/TIR-33_Center_of_Pressure.pdf

[15] Nakka, Richard. (2008). Richard Nakka's Experimental Rocketry. Disponible en: http://members.aol.com/nonillion/sucrose.html

[16] Freescale Semiconductor (2010). ACCELERATION SENSORS. Disponible en: http:// www. freescale.com/webapp/sps/site/prod_sumary.jsp?code=MMA7261QT\&fsrch=1

[17] Microchip (2010). Microcontrollers 8-bit, 40 Pins. Disponible en: http://ww1.microchip. com/downloads/en/DeviceDoc/39632e.pdf 